\title{
Reología de suspensiones de esmaltes cerámicos para monococción: estudio de la influencia de los aditivos utilizados
}

\author{
F. ANDREOLA, P. POZZI, M. ROMAGNOLI \\ Facoltà di Ingegneria, Università di Modena e di Reggio Emilia.Via Campi 183 - 41100 - Modena - Italia
}

\begin{abstract}
En este trabajo se presentan los resultados obtenidos a partir de la caracterización y el estudio del comportamiento reológico de suspensiones de fritas cerámicas para la elaboración de esmaltes para baldosas de monococción porosa. En particular se evidencian los efectos que los distintos componentes y aditivos, ejercen sobre los parámetros reológicos que controlan las fases de preparación y de aplicación. El presente estudio se ha desarrollado usando la viscosimetría rotacional como técnica de estudio, para evaluar el comportamiento reológico de los esmaltes, por medio del análisis de curvas de flujo en un amplio intervalo de viscosidades $\left(1-10^{4} \mathrm{cP}\right)$. Las pruebas reológicas ó fueron hechas en flujo continuo que aproxima el efecto de cizalla y reproduce bastante bien las condiciones de preparación y aplicación de los esmaltes.

Palabras clave: reología, esmaltes cerámicos, fritas, suspensiones

Single firing ceramic glazes: influence of the additives on the rheological behavior

In this paper are presented the results obtained by the characterisation and the study of the rheological behavior of concentrated frits suspensions used in the preparation of ceramic glazes for single firing porous tiles. In particular the effects of different components and additives on the rheological parameters which control the processing phases were put out. For this work the rotational viscosimetry has been utilised to study the rheological behavior of glaze suspensions using flow curves in a wide range of apparent viscosity values $\left(1-10^{4} \mathrm{CP}\right)$. The measurements on the glaze suspensions were carried out on continuos flow which results the best method to reproduce the conditions where the glazes are undergo during the preparation and application phases.
\end{abstract}

Key words: rheology, ceramics glazes, frits, suspensions

\section{INTRODUCCIÓN}

En la industria cerámica tradicional para la producción de pavimentos, las fases de preparación de los esmaltes resultan críticas desde un punto de vista reológico. En particular la molienda en húmedo de las materias primas y de los aditivos y la aplicación de las suspensiones de esmaltes sobre los soportes cerámicos.

Mientras el estudio del comportamiento reológico de suspensiones de arcillas cerámicas usadas en la fabricación de baldosas ha sido objeto de numerosos estudios de investigación $(1,2)$, la reología de esmaltes y de suspensiones de fritas cerámicas todavía no ha sido estudiada en profundidad, con lo que eso comporta para la obtención de baldosas esmaltadas con características estéticas de alta calidad.

Los parámetros reológicos tienen gran importancia en la definición de las condiciones de esmaltado y en el control del comportamiento reológico de los esmaltes, al momento de la aplicación que debe ser el adecuado para evitar posibles defectos superficiales (grumos, pinchados, grietas, etc.).

Los esmaltes cerámicos son suspensiones concentradas con un contenido en sólidos de $65-70 \%$ en peso. El sólido esta compuesto principalmente de un material vítreo en un
90-98 \% llamado tecnológicamente frita (producto del enfriamiento rápido en agua de un material amorfo fundido a temperaturas próximas a $\operatorname{los} 1400^{\circ} \mathrm{C}$ ) y en porcentajes no superiores al $10 \%$ de materiales arcillosos y otros aditivos. Normalmente como materiales arcillosos se utilizan, caolines o bentonitas, estos dan plasticidad a las suspensiones y evitan problemas de sedimentación. Entre los aditivos con carácter ligante figuran las carboximetilcelulosas sódicas (CMC) y como agentes dispersantes las sales sódicas de ácidos inorgánicos / orgánicos; todos estos aditivos vienen añadidos a las fritas durante la molienda.

El objetivo del presente trabajo es presentar los resultados obtenidos a partir de la caracterización y el estudio del comportamiento reológico de suspensiones de fritas cerámicas para soportes porosos, usando la viscosimetría rotacional. En particular se estudió la influencia de los componentes y aditivos utilizados, su efecto sobre los valores del esfuerzo limite de cizalla y de la viscosidad aparente.

Las pruebas reológicas fueron realizadas en flujo continuo que permiten simulan mejor las condiciones de flujo a las cuales los esmaltes vienen sometidos durante la molienda y la aplicación en los soportes. 


\section{PARTE EXPERIMENTAL}

\subsection{Preparación de las fritas utilizadas}

La frita base fue preparada a partir de un sistema ternario $\mathrm{Na}_{2} \mathrm{O} \cdot \mathrm{Al}_{2} \mathrm{O}_{3} \cdot \mathrm{SiO}_{2}$ con materias primas de uso industrial (feldespato de sodio, cuarzo, carbonatos, etc.) y composición química similar a las de las fritas de monococción para soportes porosos (3). Las composiciones derivadas se obtuvieron modificado el sistema base por la adición de un $15 \%$ en peso de óxidos modificadores. Todas las fritas fueron obtenidas por fusión, en un horno fusor semi-industrial con temperaturas entre $1420-1440^{\circ} \mathrm{C}$, después del fritado en agua, fueron secadas en estufa a $110^{\circ} \mathrm{C}$ para eliminar la humedad residual.

\subsection{Preparación de las suspensiones utilizadas}

El efecto de cada uno de los componentes del esmalte fue estudiado en cada caso con composiciones preparadas específicamente. Para evaluar la presencia del material arcilloso, se prepararon dos composiciones, una con un $95 \%$ de frita y $5 \%$ de caolín y otra con $98 \%$ de frita y $2 \%$ de bentonita. Trabajos anteriores $(3,4)$ han demostrado que las concentraciones óptimas en función de las condiciones reológicas de aplicación de los esmaltes industriales, resultan ser del $5 \%$ en peso para el caolín y del $2 \%$ en peso para la bentonita.

Para estudiar el efecto de la concentración y del grado de polimerización de los materiales ligantes presentes, fueron usadas tres carboximetilcelulosas sódicas (CMC), con diferentes grados de polimerización (alto, medio, bajo) fueron adicionadas en porcentajes variables entre $(0,1-0,5 \%$ en peso respecto al sólido seco).

TABLA I. VALORES DE LOS PARÁMETROS REOLÓGICOS OBTENIDOS MEDIANTE CORRELACIÓN DE LAS CURVAS DE FLUJO, PARA LAS DISTINTAS SUSPENSIONES PREPARADAS AL 65\% DE CONTENIDO SÓLIDO, SIN MATERIALES ARCILLOSOS Y CON: $5 \%$ EN PESO DE CAOLÍN Y $2 \%$ EN PESO DE BENTONITA.

\begin{tabular}{|l|c|c|c|c|}
\hline COMPONENTES & $\tau_{0}$ [Pa] & $\begin{array}{c}\eta_{\mathrm{p}}\left(200 \mathrm{~s}^{-1}\right) \\
\text { [mPa.s }]^{1}\end{array}$ & $\begin{array}{c}\text { nIndice } \\
\text { flujo }\end{array}$ & $\begin{array}{c}\mathbf{K} \\
\text { [mPa.s] }\end{array}$ \\
\hline FRITA & 0,10 & 16,25 & 1,10 & 10,89 \\
FRITA + 5\% CAOLIN & 0,44 & 31,05 & 1,03 & 24,16 \\
FRITA + 2\% BENTONITA & 0,00 & 56,00 & 0,98 & 58,91 \\
\hline
\end{tabular}

TABLA II. VALORES DE LOS PARÁMETROS REOLÓGICOS OBTENIDOS MEDIANTE CORRELACIÓN DE LAS CURVAS DE FLUJO PARA LAS SUSPENSIONES PREPARADAS CON: (A) $5 \%$ EN PESO DE CAOLÍN.; (B) $2 \%$ EN PESO DE BENTONITA Y 0,5\% DE CMC A DIVERSOS PESOS MOLECULARES.

\begin{tabular}{|l|c|c|c|c|}
\hline & \multicolumn{2}{|c|}{ CAOLIN } & \multicolumn{2}{c|}{ BENTONITA } \\
\hline & $\tau_{0}$ [Pa] & $\begin{array}{c}\eta_{\mathrm{p}}\left(200 \mathrm{~s}^{-}{ }^{1}\right) \\
\text { [mPa.s] }\end{array}$ & $\tau_{0}$ [Pa] & $\begin{array}{c}\eta_{\mathrm{p}}\left(200 \mathrm{~s}^{1}{ }^{1}\right) \\
\text { [mPa.s] }\end{array}$ \\
LV (BAJO PM ) & 1,10 & 179,90 & 27,60 & 342,50 \\
MV (MEDIOPM) & 1,37 & 206,40 & 44,08 & 560,80 \\
HV (ALTO PM) & 1,55 & 333,50 & 47,68 & 696,30 \\
\hline
\end{tabular}

Por su parte el efecto de los defloculantes, se estudió seleccionando tres aditivos ampliamente utilizados en el sector cerámico industrial: el metasilicato de sodio $\left(\mathrm{Na}_{2} \mathrm{O} / \mathrm{SiO}_{2}: 1 / 1\right)$; el tripolifosfato de sodio $\left(\mathrm{Na}_{5} \mathrm{P}_{3} \mathrm{O}_{10}\right)$ y el poliacrilato de sodio (PM:2100). Para la realización de las curvas de defloculación a las suspensiones preparadas con frita y materiales arcillosos, se adicionaron $0,5 \%$ en peso (respecto al sólido seco) de CMC de peso molecular medio y los agentes defloculantes en porcentajes entre 0,1 y $1 \%$ (respecto el sólido seco).

En todos los casos las suspensiones fueron preparadas al $65 \%$ en peso de sólido, en agua destilada usando un molino rápido de laboratorio con bolas de alúmina compacta durante dos horas para obtener una suspensión estable para la realización de las pruebas reológicas $(4,5)$.

\subsection{Técnicas reométricas}

Las suspensiones de esmaltes cerámicos son sistemas no Newtonianos como la mayor parte de las suspensiones altamente concentradas (4). La viscosidad resulta una función de las condiciones de flujo, no es constante y depende de los valores del gradiente de velocidad $(\dot{\gamma})$ y del esfuerzo aplicado $(\tau)$ en cada instante, y de la historia de deformación precedente al momento de la medición (6). Para este tipo de sistemas los parámetros experimentales que definen el comportamiento reológico son el esfuerzo límite de fluidez $\left(\tau_{0}\right)$, la viscosidad aparente (a un gradiente de velocidad dado) $\left(\eta_{\dot{\gamma}}\right)$ y la dependencia con el tiempo (tixotropia, anti-tixotropia). Para estudiar la reología se usó un ciclo que permitió estandardizar las mediciones e independizarse de las características tiempo dependientes de las muestras. El ciclo utilizado tiene 5 segmentos, el primero contempla una rampa de $30 \mathrm{~s}$. de duración al máximo gradiente de velocidad $\left(200 \mathrm{~s}^{-1}\right)$, para eliminar en las muestras los efectos de la fase de preparación, en el segundo las muestras permanecen en reposo durante $1 \mathrm{~min}$. Los segmentos con variación del gradiente de 0 a $200 \mathrm{~s}^{-1}$ en $10 \mathrm{~min}$. sirven para determinar las curvas di flujo, mientras que el segmento intermedio con gradiente de velocidad constante $200 \mathrm{~s}^{-1}$ durante $10 \mathrm{~min}$. es útil para dar una rápida indicación del efecto del tiempo (4). Todas las pruebas reológicas fueron realizadas en condiciones de temperatura constante a $20^{\circ} \mathrm{C}$ con un viscosímetro rotativo CV20 Haake con cilindros coaxiales ZA30 como sistema de medición.

\section{RESULTADOS Y DISCUSIÓN}

\subsection{Influencia del tipo de material arcilloso utilizado}

Las suspensiones concentradas de fritas cerámicas en agua resultan sistemas no Newtonianos, con tendencia a la dilatancia a altos gradientes de velocidad y dependientes del tiempo (3). La necesidad de aumentar la estabilidad de las suspensiones, limitando la sedimentación obliga a añadir en la composición de los esmaltes, componentes que aumentan la plasticidad. Los más utilizados resultan ser los materiales arcillosos, estos siendo partículas cargadas superficialmente, ejercen fuertes interacciones con el medio acuoso y con las partículas de frita reduciendo la movilidad del medio y consecuentemente la sedimentación (7).

En la Figura 1 se puede observar la cinética de sedimentación para suspensiones de fritas preparadas con y sin material arcilloso. Las velocidades de sedimentación fueron obtenidas con mediciones de reflectancia ( $R \%$ ) en función del tiempo, 
usando la espectrofotometría UV en el intervalo 550-650 $\mathrm{nm}$ (3). La presencia de material arcilloso produce un aumento de la estabilidad en el sistema, disminuyendo las posibilidades de sedimentación, como se evidencia en la figura estos sistemas alcanzan el equilibrio después de 6-7 hs. A diferencia de las suspensiones preparadas solamente con fritas donde no se alcanza el equilibrio ni después de 24 hs.

En particular el caolín, debido a su estructura laminar, en suspensión acuosa exhibe dos zonas con diferente carga superficial, las caras y los bordes, que ejercen fuerzas de tipo electrostático de atracción y de repulsión dando lugar, en función del pH del medio, a fenómenos de floculación y defloculación con variaciones en la viscosidad. La adición de caolín a la suspensión de fritas determina que la reología de las suspensiones sea gobernada por el carácter coloidal derivado de la arcilla, siendo las fritas materiales no plásticos y con granulometria mayor. El efecto de las fuerzas electrostáticas en la fase dispersa, reduce las colisiones entre las partículas sometidas a flujo mejorando el comportamiento reológico de la suspensión.

En el caso de adicionar bentonitas al sistema, al efecto coloidal se suma la formación de un gel tridimensional, con estructura abierta, que deriva de la interacción de la doble capa de carga opuesta sobre las caras y los bordes de las partículas (7). Esto se debe especialmente al espesor (distancia entre estratos) de las partículas de bentonita mucho mayor que las de caolín, que permite la adsorción de moléculas de agua del medio, con un aumento de la fracción volumétrica a igual contenido del sólido, aumentando la viscosidad. Este fenómeno está ligado a un proceso de reconstrucción estructural que sucede en tiempos mayores respecto a los de la medición y por eso no se evidencia en los valores del esfuerzo crítico de cizalla.

En la Tabla 1 se indican los parámetros reológicos $\left(\tau_{0}\right),(\eta a)$, $\mathrm{n}, \mathrm{K}$ de las suspensiones preparadas con caolín y bentonita, obtenidos correlando las curvas de flujo con el modelo de Herschel-Buckley(8). Del análisis de los valores presentados resulta que la presencia de caolín produce un incremento del esfuerzo límite de fluidez $\left(\tau_{0}\right)$, y la presencia de bentonita aumenta en manera marcada la viscosidad aparente $(\eta a)$ Teniendo en cuenta las diferencias observadas se puede concluir que es distinto el efecto producido según el tipo de material arcilloso utilizado. Como se ha visto además de disminuir

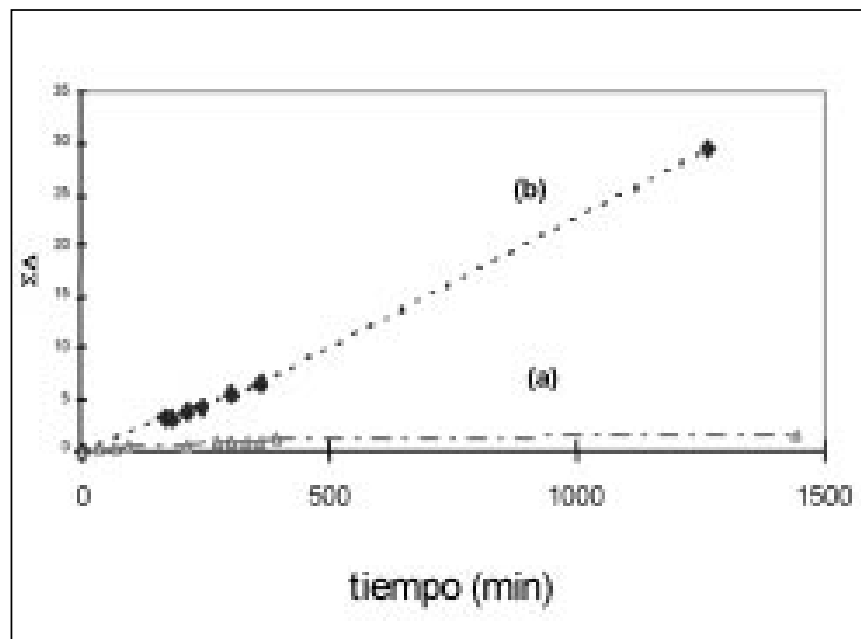

Fig. 1. Velocidades de sedimentación de suspensiones, preparadas al $65 \%$ en peso con: (a) material arcilloso y (b) sin material arcilloso. Variaciones de R\% vs. tiempo. la tendencia a la sedimentación, reducen el efecto dilatante justificado en los valores calculados del índice de flujo (n).

\subsection{Influencia del material ligante}

Los materiales ligantes usados en el sector de los esmaltes cerámicos son las carboximetilcelulosas (CMC), estas desde un punto de vista químico son polímeros hidrosolubles de tipo anionico, generalmente vienen comercializadas las sales sódicas, en cuanto esas se obtienen con mayor facilidad y poseen una elevada solubilidad en agua.

Los esmaltes, constituidos prevalentemente por compuestos no plásticos (fritas, óxidos, pigmentos, etc.) necesitan haber en su formulación materiales ligantes, como las carboximetilcelulosas, porque estas favorecen junto con los materiales arcillosos la plasticidad y la adhesión de las partículas de esmalte al soporte, en modo particular en las composiciones de monococción rápida donde las características estéticas requeridas impiden la utilización de grandes cantidades de materias arcillosas (9).

El efecto del grado de polimerización (largo de las cadenas) de las CMC añadidas fue estudiado y se puede observar en las figuras siguientes, donde se representan las curvas de flujo de

a)

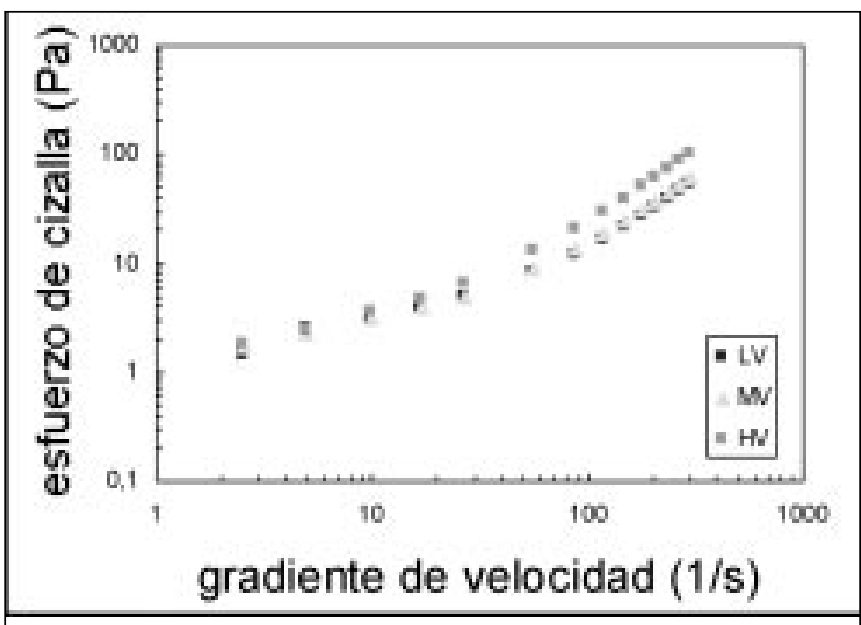

b)

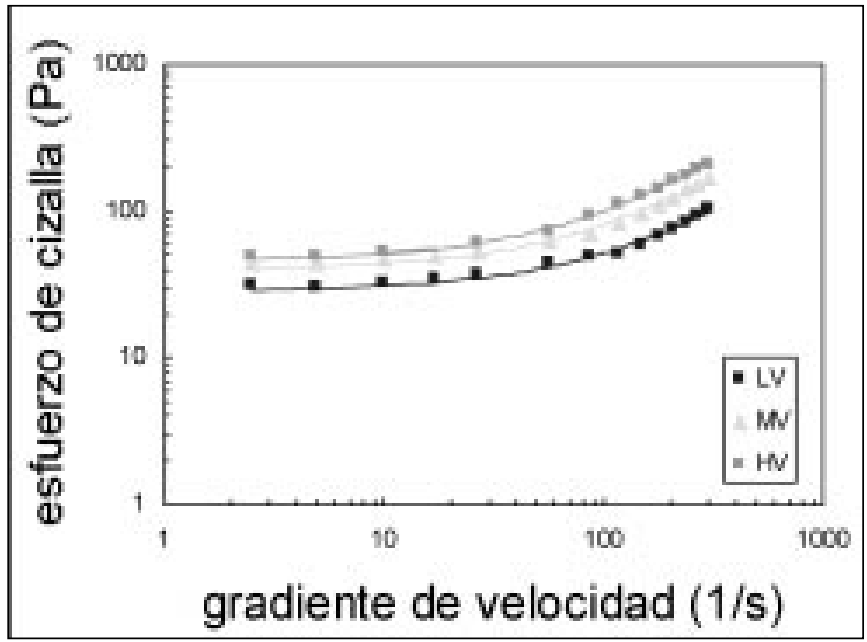

Fig. 2. Curvas de flujo (log-log) de suspensiones de esmaltes al 65\% en peso, preparadas con: (a) $5 \%$ caolín ; (b) $2 \%$ bentonita y $0,5 \%$ de $\mathrm{CMC}$ a diversos pesos moleculares. 
las suspensiones preparadas con CMC a distintos pesos moleculares. A partir del análisis de las curvas, se observa que existe una relación directa entre el peso molecular de las CMC adicionadas y la viscosidad resultante de las suspensiones. El límite de fluidez no resulta muy influenciado con la presencia de CMC en las suspensiones con caolín (figura 2a), sin embargo, en las suspensiones con bentonita el aumento es importante (figura 2b). Este distinto comportamiento puede ser imputable a dos efectos combinados, el aumento de plasticidad que provoca la CMC y el grado de gelificación de la bentonita (7). Además fue verificado que a bajas concentraciones $(0,1 \%)$ las CMC son en grado de desarrollar una acción defloculante (3).

Los valores de los parámetros reológicos, obtenidos por correlación de las curvas vienen presentados en la Tabla 2. Es de señalar que el comportamiento reológico en flujo de estos sistemas no cambia con la adición de pequeñas cantidades de CMC siendo de tipo plástico en todos los casos y con tendencia a la dilatancia a gradientes de velocidad elevados.

a)

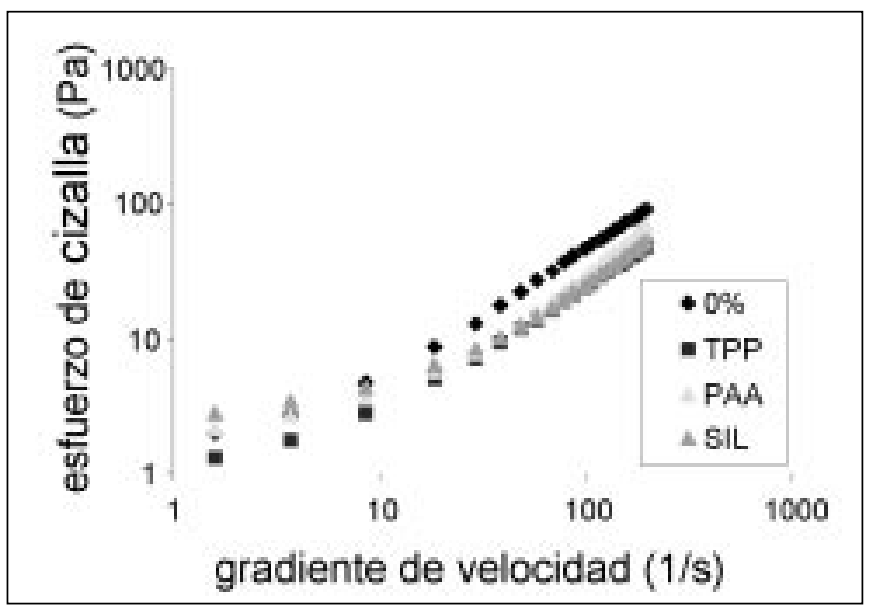

b)

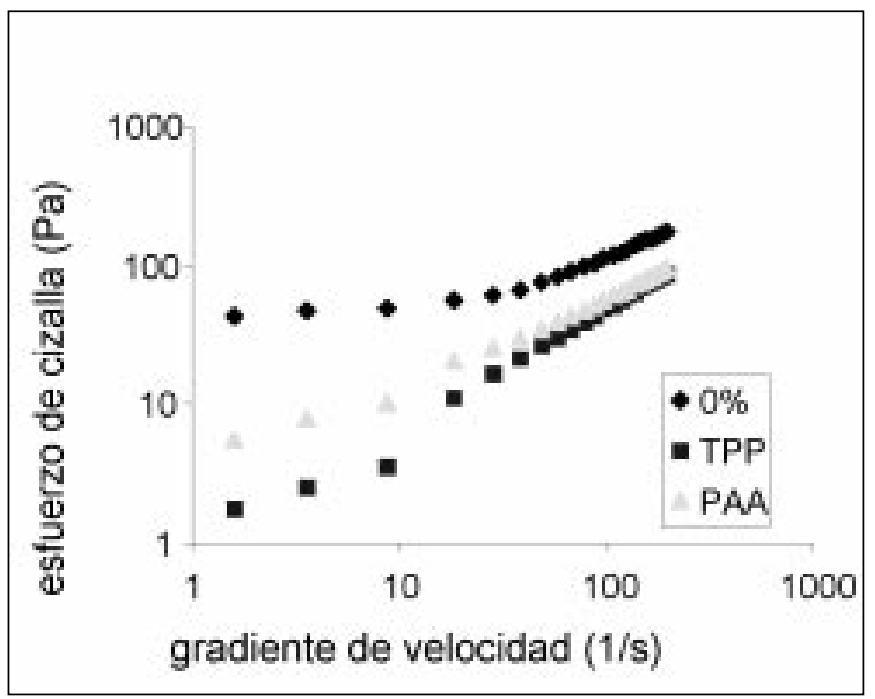

Fig. 3. Curvas de flujo (log-log) de suspensiones de esmaltes al $65 \%$ en peso, preparadas con (a) 5\% caolín + 0,5\% CMC MV; (b) 2\% bentonita $+0,5 \%$ CMC MV usando diferentes defloculantes.

\subsection{Influencia de los defloculantes utilizados}

En las suspensiones de esmaltes, las partículas de fritas desarrollan una carga superficial (ionización) como consecuencia del proceso de corrosión que sufren con el medio acuoso (10). Los iones que pasan a la solución modifican el equilibrio de las fuerzas electrostáticas existentes y el espesor de la doble capa eléctrica, provocando fenómenos de floculación o defloculación según exista predominio de fuerzas atractivas o repulsivas (7) (potencial $\zeta$ ). Existen sustancias que presentes en pequeñas concentraciones permiten regular estos fenómenos, determinando una mayor fluidez y disminuyendo la viscosidad de las suspensiones, sin tener que variar el contenido del sólido $(11,12)$.

Los tres defloculantes seleccionados se ubican entre los más usados en el sector cerámico industrial, dos de tipo inorgánico, el metasilicato de sodio (SIL) y el tripolifosfato de sodio (TPP) y uno de tipo orgánico el poliacrilato de sodio (PA). Estas sustancias operan con diferentes mecanismos en función del principio activo y de la naturaleza química (coloide protector, aumento del pH, formación de complejos, adsorción superficial, etc.), aumentan el potencial $\zeta$, actuando como dispersantes y fluidificantes $(13,14,15)$.

Las curvas de flujo de las figuras 3 a y $3 b$ corresponden a suspensiones de esmaltes preparados con caolín y con bentonita respectivamente y con los defloculantes, añadidos a la óptima concentración (correspondiente al mínimo de la viscosidad aparente de la curva de defloculación). Analizando las curvas emerge que la presencia de estos polielectrolitos en las suspensiones preparadas con caolín, no modifica en modo importante los valores del esfuerzo límite de fluidez, en cambio, provoca la disminución de la viscosidad a elevados gradientes de velocidad (Figura 3a).

Las curvas de flujo correspondientes a las suspensiones preparadas con bentonita, evidencian una disminución marcada de la viscosidad aparente y del esfuerzo límite de fluidez (Figura $3 b$ ). El metasilicato de sodio resultó ineficaz como defloculante, por ello no se presentan las curvas correspondientes (las suspensiones resultantes no pudieron ser medi-

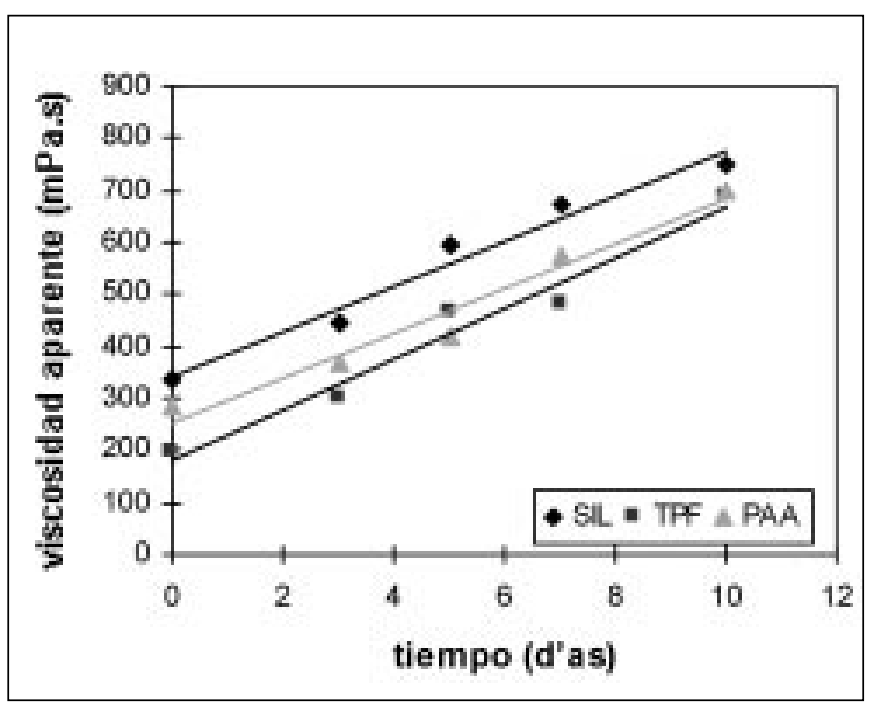

Fig. 4. Curvas de viscosidad aparente (a 200s-1) (cP) vs. tiempo de almacenamiento (días) para suspensiones de esmalte al $65 \%$ de sólidos, preparadas con $95 \%$ frita, 5\% caolín, 0,5\% CMC MV y distintos defloculantes a la concentración óptima. 
das con el viscosímetro). Además emerge, que la presencia de agentes defloculantes no cambia el comportamiento ó de las suspensiones que se mantiene de tipo plástico en todos los casos con variaciones de los parámetros reológico. Concluyendo en ambos casos el TPP resultó ser el mejor agente defloculante presentando valores de viscosidad más bajos respecto a los otros polielectrolitos considerados.

Los tres defloculantes adicionados en concentraciones superiores al valor óptimo(fenómeno de sobredefloculación), no presentan importantes aumentos de viscosidad, en cambio resulta importante el aumento del límite de fluidez. De estos resultados emerge que si el defloculante viene adicionado al momento de la aplicación del esmalte en línea, no provoca variaciones importantes de la viscosidad, permitiendo aumentar el esfuerzo límite $\left(\tau_{0}\right)$ resultando útil en algunas técnicas de aplicación (aplicación a disco).

Los esmaltes permanecen normalmente en reposo después de la molienda por algunas horas (para eliminar las burbujas de aire) y a veces días en almacenaje, antes de ser usados en las líneas de esmaltado. Para estudiar este fenómeno fueron realizadas pruebas reológicas en el arco de una semana. Las suspensiones fueron preparadas con los tres defloculantes anteriormente citados a la concentración óptima, en modo tal de garantir la estabilidad (bien dispersas) y se comparó el efecto en función del aditivo utilizado. En la figura 4 se presentan los resultados obtenidos, se puede apreciar que el efecto del tiempo es importante en todos los casos, las suspensiones sufren un aumento de la viscosidad aparente en función del tiempo. Con un efecto más marcado en el caso de las suspensiones que usan el TPP. Se puede suponer que la concentración óptima de defloculante, necesaria para tener el sistema disperso durante la molienda, no sea suficiente para evitar la reagrupación de las partículas de frita y material plástico cuando están en reposo o sujetas a bajos esfuerzos (tanques de agitación).

\section{CONCLUSIONES}

Los materiales arcillosos añadidos en las suspensiones de fritas cerámicas, provocan un cambio en el comportamiento reológico de las mismas de dilatante a pseudo-plastico usando bentonitas o plástico usando caolines. La presencia de carboximetilcelulosas usadas como materiales ligantes, no cambia el comportamiento reológico de las suspensiones, resultando de tipo plástico en todos los casos. Se observa un aumento de la viscosidad aparente en función del peso molecular y efectos diferentes sobre el esfuerzo límite de fluidez en función del material arcilloso presente. El empleo de agentes defloculantes, resulta necesario para optimar la reología de los esmaltes durante la molienda y en algunos casos para corregir los parámetros reológicos al momento de la aplicación en línea. De las pruebas reológicas realizadas emerge que la presencia de estos aditivos no cambia el comportamiento reológico de las sus- pensiones que se mantiene de tipo plástico, en todos los casos con variaciones de los parámetros reológicos. Independientemente del material arcilloso presente en el esmalte el TPP dió los mejores resultados, presentando valores de viscosidad más bajos respecto a los otros polielectrolitos considerados.

En base a los resultados obtenidos se concluye que usando la viscosimetría rotacional como técnica de control y de estudio para esmaltes industriales, esta permite conocer en modo anticipado los efectos que sobre la reología ejercerán los distintos componentes y aditivos usados en la formulación de los esmaltes. Permitiendo la racionalización y la optimación de la composición más adecuada para obtener los parámetros reológicos requeridos en las distintas técnicas de esmaltado que vengan usadas (campana, disco, aerosol, etc.).

\section{BIBLIOGRAFIA}

1.T.Manfredini, G.C. Pellacani, P.Pozzi, A.Blasco Fuentes, F.Negre Medall, “Some General Considerations on the Rheological Behaviour of Aqueous Clay Suspensions: Dependence of Physico-Chemical Properties of Inorganic Salts, Calcium(II) Ion presence and Grinding Times", Ind. Ceram., 9, [2] 58-62 (1989).

2.T.Manfredini, G.C.Pellacani “Indagini reologiche su sospensioni di argille per la ottimizzazione di processi di deflocculazione " Ceramurgia, XVI, (1) 30-33 (1986).

3. F.Andreola, "Caratterizzazione e Studio del comportamiento reologico di sospensioni di fritte e smalti ceramici per supporti ad elevata porosità" Tesis de Doctorado en Ingeniería de los Materiales, Università di Modena, (1997).

4. F.Andreola, A.Bonamartini Corradi, T. Manfredini, G.C. Pellacani, P.Pozzi, “ Concentra ted glaze suspensions" Am. Cer. Soc. Bull.73[10] 75-78 (1994).

5. F.Andreola, P.Pozzi, M. Romagnoli, "Wet-Grinding Ceramics Frits: Effects of the size grinding media, grinding time and solid content", Mat. Eng., 8, (2) 171-180 (1997).

6. R.Lapasin, "Introduzione alla reologia" in" La reologia dei materiali ceramici tradizionali", Ed. P.Pozzi, C.Galassi, Faenza Editrice, p.p 1-27 (1994).

7. C.Galassi, "Proprietà chimico-fisiche e caratteristiche strutturali di sistemi ceramici", in" La Reologia dei materiali ceramici", p.p 67-92, Faenza Editrice, (1994).

8. R.Tanner, "Engineering Rheology, Non-Newtonian fluid behaviour in shear", Oxford Eng. Science Vol 14 11-18 (1988).

9. D. Chiavacci, " I polielettroliti sintetici nella smaltatura. Criteri di scelta, evoluzione e sviluppo", Cer. Inf., 335, (10) 593-599 (1995).

10. R.G. Newton, " Durability of glass, a review", Glass technology, 26,(1) 21-38 (1985).

11. Q. Malandrino, "I fenomeni elettrocinetici . Deflocculazione e Flocculazione", Cer. Inf. 124 (11) 53-60 (1976).

12. Q. Malandrino, "Gli additivi nell'Industria Ceramica: i deflocculanti", Cer Inf. 154 (3) 135-141 (1979).

13.T.Manfredini, P.Pozzi ed altri, "Silicati di sodio come agenti deflocculanti", Ceramurgia,17 (5) 183-85 (1987).

14. T.Manfredini, P.Pozzi ed altri, "Monomeric and oligomericphosphates as deflocculant.", Applied Clay Science (5) 193-201 (1990).

15. A. Bonamartini,T. Manfredini ed altri, "Deflocculation of concentrated aqueous clay suspensions with sodium polymethacrylates", J.Am.Ceram.Soc, 77 (2) $509-13$ (1994)

Recibido: 10-11-98

Aceptado: 1-3-99 